\title{
Does Patient's Self-Awareness Predict Ankle Fractures?
}

\author{
Shaza Aouthmany ${ }^{a}$ Tymon Horn ${ }^{a}$ Mahesh R. Pillai ${ }^{b}$ Edward J. Kakish ${ }^{c}$ Alana M. Kakish ${ }^{d}$
}

Coresponding author(s): shaza.aouthmany@utoledo.edu

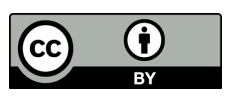
${ }^{a}$ Department of Emergency Medicine, University of Toledo Medical Center, Toledo, OH 43614, USA, ${ }^{b}$ Human Research Protection Program, University of Toledo
Medical Center, Toledo, OH 43614, USA, ${ }^{c}$ Emergency Departmen, John D. Dingell VA Medical Center, Detroit MI 48201, USA, and ${ }^{d}$ Michigan State University, East
Lansing MI 48824, USA

Introduction: Ankle injuries represent one of the most common sports and extremity related complaints presented in emergency departments (ED) with over five million ankle injuries annually arising in the United States. The 2007 US National Health Statistics Report stated that lower limb and ankle complaints accounted for $4.1 \%$ of all reported body sites undergoing injury in the ED. The purpose of this study was to examine how frequently a sample of ED patients' perception of having a possible ankle fracture was predictively associated with results of their X-ray evaluation results. Methods: After 2017 IRB approval, a sample of consented adult patients receiving care at the authors' two Ohio and Michigan ED were asked, "Do you think you broke your ankle?" Regardless of each patient's answer, they received an $X$-ray to evaluate for an ankle fracture that was interpreted by a radiologist. Results: A total of 69 eligible patients received an ankle X-ray. The total number of ankle fractures confirmed by Xray was $20(29.4 \%$ of sample) while $48(70.5 \%)$ of ankles that were X-rayed were not fractured. Six $(28.5 \%)$ out of 21 males and 14 $(30.4 \%)$ out of 46 females were found to have a confirmed ankle fracture. The sensitivity of the perceived ankle fracture question was at or below $\mathbf{5 0 \%}$ in all sample subgroups except for smokers at $67 \%$. Conclusion: These findings support the importance of an ED clinician's intuition when considering a patient's own selfassessment during their clinical decision-making processes

X-ray | emergency department | ankle | fracture | radiation | healthcare cost I pain scale I ambulation status I self-awareness

nkle injuries represent one of the most common sports and extremity related complaints presented in emergency departments (ED), with over five million ankle injuries arising annually in the United States $(1,2)$. The 2007 US National Health Statistics Report stated that lower limb and ankle complaints accounted for $4.1 \%$ of all reported body sites incurring injury in ED (3). The incidence of ankle injuries presenting in other clinic and athletic settings (4).

Although many such injuries of the ankle and foot (includes ligament and tendon impairment, forms of impingement, lesions, and stress-related fractures) can result from overuse behaviors, the need for imaging continues to a key avenue to ankle injury management (5). The criteria for ordering ankle X-ray is a topic that warrants further conversation (6). The Ottawa ankle rules have become commonplace practice for ankle injury management, providing a widely validated framework for use by healthcare professionals $(7,8)$. Collectively, the rules account for a patient's pain locale and weight bearing capability of the injured ankle immediately following injury in an ED. The original Ottawa ankle study findings have been validated in large, multi-center studies (9).

Other types of clinical rules, with a sensitivity of $100 \%$ and a specificity of $36 \%$ for patients with recent ankle injuries can help determine which ankle injuries warrant $\mathrm{X}$-ray imaging to rule out ankle fracture (10). Unfortunately, alternative methods have not provided comparable observations between patients and clinicians, and teaching the clinical rules to patients has not proven effective in reducing ED visit frequency (7).

The healthcare burden of undergoing an X-ray for an ankle injury has remained a discussion point, with the risk and benefit analysis visited during management (11). With rising healthcare costs, limiting unnecessary radiographic studies is one way to decrease

Submitted: 08/06/2020, published: 20/01/2021.

Freely available online through the UTJMS open access option 
healthcare provision costs.

Although the conservative approach is to X-ray the injured ankle, it has been shown that less than $15 \%$ of all ankle injuries have actually resulted in a fracture confirmed by X-ray (12). The risk of radiation exposure also must be weighed in the decision-making process (13).

The radiation exposure from a combined anterior-posterior and lateral ankle X-ray is $0.0015 \mathrm{mSv}$ (13). To put this into perspective, the average person in the US is annually exposed to $3.0 \mathrm{mSv}$ of background radiation (14). There is epidemiological evidence to show that acute exposure to greater than $10-50 \mathrm{mSv}$ of radiation increases risk of some cancers (15). However, researchers have had difficulty quantifying cancer risks to acute exposures less than $10 \mathrm{mSV}$, which does not necessarily imply that these very small doses of radiation are negligible.

Even if there are relatively minor cancer risks imposed from imaging, this can result in a significant public health concern secondary to the frequency and overall quantity of X-ray imaging (16).

Purpose of Study The purpose of this study was to examine the potential benefit a pa-tient's perception can have on healthcare providers' decision whether patients require X-rays and which types of patients should just be clinically observed. This study team wished to correlate the utility of X-ray evaluation based on patients' perspectives of ankle fracture using a prospective non-randomized cross-sectional descriptive study design. The null hypothesis of the authors was that patient perceptions of ankle fracture would have no significant relationship on X-ray decision-making processes of sample ED providers.

\section{Materials and Methods}

After obtaining 2017 IRB approval, data were collected from an adult patient sample of participants obtaining ED workup for possible ankle fracture at the ED of the University of Toledo Medical Center in Toledo, $\mathrm{OH}$ and St. Mary Mercy Hospital in Livonia, MI. Sampling exclusion criteria consisted of the following: any other distracting injury, inability to answer the question posed, any patient who had not undergone a radiographic evaluation for their ankle complaint, any non-English speaker, pregnant women and prisoners.

Patients who presented to the ED with an ankle injury complaint were first asked if they would like to participate in a study that involved answering one simple question. If they agreed, they were presented with an IRB-approved informed consent form to sign. After consent was obtained, a researcher (i.e., attending physician or resident) asked the pa-tient "Do you think you broke your ankle?" No data were obtained for any patient who could not complete this process. Regardless of the answer, the patient received an X-ray to look for an ankle fracture based on the clinician's decision-making process.

After the X-ray was obtained and interpreted by a radiologist, the diagnosis of ankle fracture or no ankle fracture was compared to the patient's original verbal answer. The radiologist's interpretation of the X-ray was observed as the gold standard for diagnos-ing ankle fractures. The research team later populated a paper data sheet form the chart with the patient's response and demographic information.

The sources of data were from the patient directly or the medical records. Participants were randomly assigned a study number, which protected their confidentiality. All data were maintained on a secured drive and only members of the study team, the Principal Investigator (PI Edward Kakish), co-investigators and the associates on the team had access to study data. The PI and research coordinator trained all of the chart abstractors in the proper protocol for medical record data abstraction. Data concerning the fol-lowing variables were collected: gender, race, smoking status, ambulatory status and pain score.

\section{Results}

A total of 69 patients who met inclusion criteria provided informed consent. Of the 69 patients evaluated, $47(68.1 \%)$ were female, $21(30.4 \%)$ were male with one $(1.4 \%)$ patient's sex not obtained (Table 1). Only 46 females were included in the analysis as the X-ray result of one patient was not recorded.

The total number of ankle fractures confirmed by X-ray was 20 (29.4\%) while $48(70.5 \%)$ of ankles that underwent an X-ray were not fractured (Table 1). The total number of males who fractured their ankle was six $(28.5 \%)$ out of 21 total who underwent X-ray (Table 1). The total number of females who fractured their ankle was $14(30.4 \%)$ out of 46 total who underwent X-ray. (Table 1). The one sample patient whose sex was not recorded was not found to have fractured their ankle.

The racial affiliation and demographic characteristics of sample patients were as follows: $35(50 \%)$ of white descent, five (7\%) of African American descent, seven $(10 \%)$ were either unknown or declined to answer and the remaining $22(32 \%)$ participants were of Hispanic or Asian descent.

The predictive performance of the initial question, "Do you think you broke your ankle?" was evaluated as a screening measure, with the radiologist's interpretation of the ankle X-ray observed as the gold standard for diagnosing ankle fractures. The sensitivity of the question was at or below $50 \%$ in all sample subgroups with the exception of smokers at $67 \%$. (Table 2 ). The specificity of all patients was $67 \%$, which increased to $79 \%$ in patients who were able to ambulate without assistance. (Table 2).

Overall, the positive predictive value (PPV) of the question posed was $30 \%$ while the false discovery rate (FDR) was $70 \%$ (Table 3). The negative predictive value (NPV) of the question passed was $71 \%$ while the false omission rate (FOR) was $29 \%$. (Table 3 ). The female and male patient statistics were separated to elucidate any potential discrepancies between the sexes. In total, of the $45 \mathrm{fe}-$ males included, the PPV was $31 \%$ while the FDR was $69 \%$. (Table 3 ). Of those females who answered "No", the NPV was $70 \%$ while the false omission rate (FOR) was $30 \%$. (Table 3). Of the 21 total male participants included, the PPV was $33 \%$ while the FDR was $67 \%$ (Table 3). Of those men who answered "No", the NPV was $73 \%$ and the FOR was $27 \%$. (Table 3 ).

In addition to stratifying patient data by gender, Table 3 includes the following terms: smoking status and ambulatory status. Smokers and non-smokers had a PPV of $33 \%$ and $29 \%$ respectively and the NPV was $67 \%$ and $73 \%$ respectively. (Table 3.) A total of 22 patients were non-ambulatory in the ED with a PPV of $55 \%$ and a NPV of $46 \%$. (Table 3). Furthermore, a total of 27 patients were ambulatory without assistance in the ED. The PPV and NPV of this ambulatory sample subgroup was $17 \%$ and $91 \%$ respectively (Table $3)$. 
Table 1. Patient X-ray Results

\begin{tabular}{|c|c|c|c|c|}
\hline & All Patients & Male & Female & Unknown Sex \\
\hline Fracture & $20(29.4 \%)$ & $6(28.5 \%)$ & $14(30.4 \%)$ & $0(0 \%)$ \\
\hline No fracture & $48(70.5 \%)$ & $15(71.5 \%)$ & $32(69.6 \%)$ & 1 \\
\hline Total Evaluated by X-ray & 68 & 21 & 46 & 1 \\
\hline
\end{tabular}

Table 2. Sensitivity and Specificity of Question "Do you think you broke your ankle?" as a screening test

\begin{tabular}{ccccccccc}
\hline \hline & $\begin{array}{c}\text { All } \\
\text { Patients }\end{array}$ & Male & Female & Smokers & Nonmokers & $\begin{array}{c}\text { No } \\
\text { ambulation }\end{array}$ & $\begin{array}{c}\text { Ambulation } \\
\text { with assistance }\end{array}$ & $\begin{array}{c}\text { Ambulation } \\
\text { without assistance }\end{array}$ \\
\hline $\begin{array}{c}\text { Sensitivity } \\
\text { Specificity }\end{array}$ & $35 \%$ & $33 \%$ & $36 \%$ & $67 \%$ & $27 \%$ & $50 \%$ & $0 \%$ & 33 \\
& $67 \%$ & $73 \%$ & $66 \%$ & $33 \%$ & $74 \%$ & $50 \%$ & $62 \%$ & $79 \%$ \\
\hline
\end{tabular}

Table 3. Table 3. Patient Accuracy When Responding Yes or No to the Question Do you think you broke your ankle?

\begin{tabular}{ccccccccc}
\hline \hline & $\begin{array}{c}\text { All } \\
\text { Patients }\end{array}$ & Male & Female & Smokers & Nonmokers & $\begin{array}{c}\text { No } \\
\text { ambulation }\end{array}$ & $\begin{array}{c}\text { Ambulation } \\
\text { with assistance }\end{array}$ & $\begin{array}{c}\text { Ambulation } \\
\text { without assistance }\end{array}$ \\
\hline PPV & $30 \%$ & $33 \%$ & $31 \%$ & $33 \%$ & $29 \%$ & $55 \%$ & $0 \%$ & $17 \%$ \\
FDR & $70 \%$ & $67 \%$ & $69 \%$ & $67 \%$ & $71 \%$ & $46 \%$ & $100 \%$ & $83 \%$ \\
NPV & $71 \%$ & $73 \%$ & $70 \%$ & $67 \%$ & $73 \%$ & $46 \%$ & $62 \%$ & $90 \%$ \\
FOR & $29 \%$ & $27 \%$ & $30 \%$ & $33 \%$ & $28 \%$ & $55 \%$ & $39 \%$ & 10
\end{tabular}

Positive Predictive Value, FDR = False Detection Rate, $N P V=$ Negative Predictive Value, FOR = False Omission Rate 
Table 4. Pain score mean in patients with and without ankle fracture

\begin{tabular}{ccc}
\hline \hline & $\begin{array}{c}\text { Pain Score } \\
(\mathbf{1 - 1 0})\end{array}$ & $\begin{array}{c}\text { 95\% Confidence } \\
\text { Intrval }\end{array}$ \\
\hline Fracture & 6.5 & $5.2-7.7$ \\
No fracture & 6.9 & $6.2-7.7$ \\
\hline
\end{tabular}

A 10-point pain scale was also administered to patients with 1 being Ino pain" and 10 being the lworst pain ever". The mean pain score for those who fractured their ankle was 6.5 (95\% CI 5.2-7.7) while the mean pain score for those who did not fracture their ankle was 6.9 (95\% CI 6.2-7.7). (Table 4).

\section{Discussion}

Ankle injuries are among the most common patient complaints in the ED settings (1). This study aimed to examine the predictive association of patients' perceptions of a possible ankle fracture. $\mathrm{Pa}$ tients were asked, IDo you think you broke your ankle?", and their answers were compared to their final ankle X-ray results verified by a radiologist. Descriptive analyses were performed to investigate how often a sample patients' answers matched imaging results during their clinical evaluations.

The sensitivity of the screening question "Do you think you broke your ankle?" was generally low and was less than or equal to $50 \%$ among all groups except smokers $(67 \%)$. The question's low sensitivity demonstrates that it was a relatively poor screening measure to rule out an ankle fracture. However, its specificity was greater among most groups and highest among patients who were ambulatory without assistance $(79 \%)$, non-smokers $(74 \%)$, and males (73\%). Therefore, this screening question's higher specificity demonstrates it could be somewhat helpful for to ruling ankle fractures, particularly among ED patients who are ambulatory without assistance.

Based on the low demonstrated PPV (30\%), patients' perceptions of their possible an-kle fracture was not generally reliable and most patients (i.e, 70\%) incorrectly thought that they had sustained an ankle fracture. However, the high NPV (71\%) showed a stronger association with the lack of an ankle fracture diagnosed by X-ray. Thus, if ED patients stated during this study that they did not think they had an ankle fracture, they were more likely to be accurate when confirmed by X-ray.

Of the different patient characteristics analyzed, a patient's ambulatory status was no-tably associated with a patient answering "Yes" to the screening question confirmed by X-ray. The PPV of this question was the highest for patients who were non-ambulatory $(55 \%)$, compared to patients who were ambulatory with and without assistance ( $0 \%$ and $17 \%$ respectively).

Additionally, the NPV was highest for patients who were ambulatory without assis-tance $(90 \%)$, compared to patients who were ambulatory with assistance (62\%) and non-ambulatory (46\%). Thus, sample patients who were ambulatory were more likely to correctly evaluate their ankle fracture, while patients who were able to ambulate with-out assistance were more likely to incorrectly perceive their ankle injury. These study findings demonstrates that patients are more likely to correctly assess their ankle injury when they have a worse ambulatory status.

Furthermore, we used a validated numerical scale pain score (17) to record and analyze patients with and without later-confirmed ankle fractures. However, in this study, the confidence intervals for pain scale ratings of both patient groups overlapped, show-ing no significant difference. Thus, these results indicate that use of numerical pain rating scales may not be as useful during clinical decision-making processes concern-ing whether to order an X-ray for a potential ankle fracture in ED settings.

Several potential limitations of this study may be that the patient data from a smaller convenience sample of ED adult patients who were stratified by only three factors (i.e., gender, smoking status and ambulatory status). Although poor ambulatory status ap-peared to be more closely associated to an accurate self-assessment ankle fracture than sex or smoking status, it is possible that this outcome may be associated with other unmeasured factors (e.g., age, socioeconomic status or educational level). Also, since this study did not account for previous ankle injuries, it is possible that patients with prior ankle injuries could have considered their past experiences when answering the pre-X-ray screening questions.

\section{Conclusion}

Patients commonly present to the ED with ankle injuries. However, considering rising healthcare costs and radiation exposure, there is a need to improve the current yield of ankle X-rays and develop better tools to aid in assessing ankle fractures. This study aimed to determine the association between ED patients' selfassessment of a possible ankle fracture and true ankle fractures diagnosed by X-ray.

\section{Conflict of interest}

Authors declare no conflict of interest. 


\section{Institutional Review Board approval}

All procedures performed involving human participants were in accordance with the ethical standards of the institutional research committee (University of Toledo Institutional Review Board, Reference IRB \# 200287).

\section{Authors' contributions}

1. Wedmore IS, Charette $\mathrm{J}$ (2000) Emergency department evaluation and treatment of ankle and foot injuries. Emerg Med Clinics No Amer 18(12): 85-113.

2. Daly PJ, Fitzgerald RH, Melton LJ, et.al. (1987) Epidemiology of ankle fractures in Rochester, Minnesota. Acta Orthopaed Scand 58.5:539-544.

3. Niska R, Farida B, Jianmin X (2010) National hospital ambulatory medical care survey: 2007 emergency department summary. Natl Health Stat Report 26:1-31.

4. Kaminski TW, Hertel J, Amendola N, et al. (2013) National Athletic Trainers' Association Position Statement: Conservative Management and Prevention of Ankle Sprains in Athletes. Journal of Athletic Training 48(4):528\{45.

5. Teh J, Suppiah R, Sharp R, et. al. (2011) Imaging in the assessment and management of overuse injuries in the foot and ankle. Sem Musculoskell Radiol 15(1):101-14

6. David S, Gray K, Russell JA, et. al. (2016) Validation of the Ottawa Ankle Rules for Acute Foot and Ankle Injuries. Journal of Sport Rehabilitation 25(1):48\{51.

7. Blackham JEJ, Claridge T, Benger JR (2008) Can patients apply the Ottawa ankle rules to themselves? Emerg Med J25(11):750-751.

8. Stiell I, Wells G, Laupacis A, et. al. (1995) Multicentre trial to introduce the Ottawa ankle rules for use of radiography in acute ankle injuries. Multicentre Ankle Rule Study Group. BMJ 311(7005):594-7.

9. Barelds I, Krijnen WP, Van de Leur JP, et. al. (2017) Diagnostic Accuracy of Clinical Decision Rules to Exclude Fractures in Acute Ankle Injuries: Systematic Review and Meta-Analysis. The Journal of Emergency Medicine 55(3):353\{368.
SA: Study concept, design project writing, and project development TH: Study concept and design, project develop EJK/MRP: Study concept and design, data collection, and data analysis; MRP/AMK: manuscript editing and revision of content. All authors wrote the manuscript, read and approved the final document. All authors wrote the manuscript, read and approved the final document.

10. Stiell IG, Greenberg GH, McKnight RD, et. al. (1992) A study to develop clinical decision rules for the use of radiography in acute ankle injuries. Ann Emerg Med 21(4):384-90.

11. Ramasubbu B, McNamara R, Okafor I, et. al. (2015) Evaluation of Safety and CostEffectiveness of the Low Risk Ankle Rule in One of Europe's Busiest Pediatric Emergency Departments. Pediatric Emergency Care 31(10):685\{687.

12. Stiell, IG, McDowell I, Nair RC, et. al. (1992) Use of radiography in acute ankle injuries: physicians' attitudes and practice. CMAJ 147(11):1671.

13. Koivisto J, Kiljunen T, Kadesjo N, et. al. (2015) Effective radiation dose of a MSCT, two CBCT and one conventional radiography device in the ankle region. $J$ Foot Ankle Res. 8(1):8

14. Wall BF, Hart D (1997) Revised radiation doses for typical X-ray examinations. Brit J Radiol 70:437-439.

15. Strauss HW (2008) Radiation Dose From Adult and Pediatric Multidetector Computed Tomography. JAMA 299(8):962-963.

16. Brenner DJ, Doll R, Goodhead DT, et. al. (2003) Cancer risks attributable to low doses of ionizing radiation: assessing what we really know. Proc Natl Acad Sci USA 100(24):13761-6.

17. Bijur PE, Clarke TL, Gallagher EJ (2003) Validation of a verbally administered numerical rating scale of acute pain for use in the emergency department." Acad Emerg Med 10(4):390-392. 